\title{
SCIENTIFIC REPORTS

\section{OPEN A Comparative Evaluation of the New Genexpert MTB/RIF Ultra and other Rapid Diagnostic Assays for Detecting Tuberculosis in Pulmonary and Extra Pulmonary Specimens}

John Osei Sekyere ${ }^{1}$ Nontobeko Maphalala ${ }^{1}$, Lesibana A. Malinga $a^{3,4}$ Nontombi M. Mbelle ${ }^{1,2}$ \& Nontuthuko E. Maningi $\mathbb{1}^{1 *}$

Studies evaluating the new GeneXpert Ultra with other rapid diagnostic assays are limited, particularly in different geographical settings. The performance of the GeneXpert Ultra, the GeneXpert G4, the Line probe assays (LPA) and auramine smear microscopy in detecting TB in pulmonary and extrapulmonary samples were thus evaluated. Remnants ( $n=205$ samples) of pulmonary ( $n=125$ samples) and extra-pulmonary ( $n=80$ samples) specimens from TB suspects were prospectively collected. Each sample was divided for diagnosis using microscopy, GeneXpert MTB/RIF assays, and LPA; these were all comparatively evaluated, using the MGIT 960 culture as a gold standard. The sensitivity and specificity of microscopy, Xpert Ultra, Xpert G4 and MTBDRplus (ver 2) in pulmonary samples were respectively: $82.00 \%$ and $90.28 \%$; $88.00 \%$ and $58.57 \% ; 79.59 \%$ and $90.28 \% ; 80.00 \%$ and $11.11 \%$. For extrapulmonary specimen, the sensitivity and specificity were respectively: $53.85 \%$ and $98.51 \% ; 69.23 \%$ and $49.25 \% ; 50.00 \%$ and $97.01 \% ; 69.23 \%$ and $25.37 \%$. The new and improved GeneXpert Ultra assay was more sensitive than GeneXpert G4 and LPA in both pulmonary and extra pulmonary samples, albeit with lower specificity than the GeneXpert G4. The auramine and LPA tests were also highly sensitive, although the LPA was less specific.

Although curable, tuberculosis (TB) remains the deadliest infectious disease caused by a single aetiological agent, Mycobacterium tuberculosis (M. tb), among all infectious diseases. In 2017, 1.5 million people died of TB globally. In South Africa, HIV-negative TB deaths were estimated to be 23000 (17000-29000) while HIV-positive mortalities totalled 101000 (67 000-142 000) in 20171,2. The WHO has reported a decline in TB incidence and mortality rates, and this has been due to the introduction of rapid diagnostic assays and effective TB treatment. Despite the global decline in new infections, the emergence of multidrug-resistant TB (MDR-TB) is worsening the burden of TB. In 2017, 558000 people had MDR-TB, an increase from 490000 people in 2016 while in South Africa, a decline from 19000 cases in 2016 to 15000 cases in 2017 was reported ${ }^{1}$. In South Africa, this decline was due to major efforts by the government to manage the problem, including the rolling out of GeneXpert machines throughout the country for quick diagnosis, screening high-risk groups (HIV infected individuals) to prevent transmission, treating latent TB, and using quality $M$. tb drugs for treatment ${ }^{3}$.

In 2010, the WHO recommended the use of the GeneXpert MTB/RIF G4 (hereafter referred to as Xpert G4), which is an automated cartridge-based molecular test, as a primary test to increase TB detection and improve diagnosis of rifampicin (RIF) resistance in pulmonary and extra-pulmonary TB (EPTB) specimens ${ }^{4}$. However,

${ }^{1}$ Department of Medical Microbiology, University of Pretoria, 0084, Pretoria, South Africa. ${ }^{2}$ National Health Laboratory Services, Tshwane Academic Division, 0084, Pretoria, South Africa. ${ }^{3}$ South African Medical Research Council, TB Platform Unit, 0084, Pretoria, South Africa. ${ }^{4}$ Department of Internal Medicine, University of Pretoria, 0084, Pretoria, South Africa. *email: nontuthuko.maningi@up.ac.za 


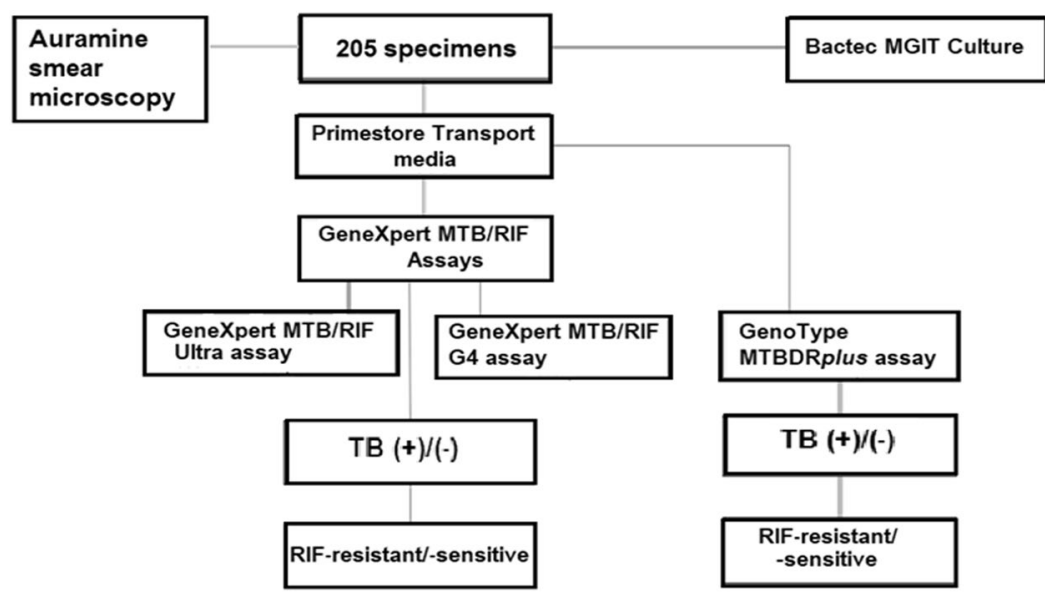

Figure 1. Flow diagram of methodologies used in processing samples and evaluating the diagnostic efficacies of smear microscopy, Xpert Ultra, Xpert G4 and LPA tests in detecting Mycobacterium tuberculosis from pulmonary and extra-pulmonary samples.

the Xpert G4 assay's sensitivity for TB detection is inadequate when few bacilli are present in the specimens, particularly in vulnerable groups such as HIV-infected patients, children and in extra-pulmonary TB samples ${ }^{5-7}$. Moreover, in terms of RIF resistance detection, the Xpert MTB/RIF G4 cartridge assay gives false-positive results for strains that carry phenotypically silent mutations (synonymous mutations), or for paucibacillary specimens $^{8,9}$. This has led to the limited usefulness of the Xpert MTB/RIF G4 cartridge assay in diagnosing TB and RIF resistance.

A new GeneXpert MTB/RIF Ultra (hereafter referred as Xpert Ultra) assay has been developed to overcome the limitations of the old Xpert MTB/RIF G4 assay with improved sensitivity in the detection of TB and RIF resistance. The Xpert Ultra assay is a rapid assay that uses an improved assay chemistry and cartridge design. It incorporates two different multicopy amplification targets viz., IS6110 and IS1081, and the RIF resistance-determining region (RRDR) of the rpoB gene ${ }^{10,11}$. These changes resulted in a ten-fold improvement in the lower limit of TB detection. Additionally, analytical laboratory data also showed an improved differentiation of certain silent mutations, improved detection of RIF resistance in mixed infections and reduced false-positive results in detecting RIF resistance in paucibacillary specimens.

Currently, only one multicentre study has been conducted to evaluate the performance of the new Xpert Ultra assay ${ }^{10}$. In that study, adults presenting with pulmonary TB symptoms at primary healthcare centres and hospitals in eight countries i.e., South Africa, Uganda, Kenya, India, China, Georgia, Belarus, and Brazil, were recruited. Reported findings showed that the sensitivity of Xpert Ultra was superior to that of Xpert G4 in patients with paucibacillary disease and in patients with HIV. However, an increase in sensitivity led to a decrease in specificity $^{10}$. Thus, more studies are needed to assess the performance of the new Xpert MTB/RIF Ultra assay on pulmonary and extra-pulmonary samples in different geographical settings.

Besides the Xpert G4, the Xpert Ultra, the auramine smear microscopy and Hain's line probe assay (LPA), which are also WHO-approved diagnostic tests for TB detection ${ }^{12}$, were compared using the BACTEC MGIT 960 culture assay as a gold standard in all cases. To our knowledge, no evaluation study has been undertaken to compare the Xpert Ultra to the smear microscopy and LPA, making this the first. This study therefore aims to provide additional and informative data on the diagnostic efficiencies of these assays to inform TB diagnostic choices, particularly in less-resourced clinical microbiology laboratories.

\section{Materials and Methods}

Study design and setting. This study prospectively evaluated and compared the TB detection capabilities of the new GeneXpert MTB/RIF Ultra assay (Cepheid Inc, USA), the old GeneXpert MTB/RIF G4 (Cepheid Inc, USA), the GenoType MTBDRplus assay (Hain Lifescience, Nehren, Germany) and the auramine smear microscopy using BACTEC MGIT 960 (BD, Sparks MD, USA) culture as a gold standard (Fig. 1).

Remnants of pulmonary (bronchial alveolar lavage $[\mathrm{BAL}]$, trachea, trachea aspirate, and sputum $)(\mathrm{n}=125)$ and extra-pulmonary (urine, superficial swabs, gastric aspirates, abscess, tissue, cerebrospinal fluid [CSF], and fluid aspirates [from non-pulmonary but unspecified/unknown anatomical site] $)(\mathrm{n}=80)$ clinical specimens were collected from the National Health Laboratory Services (NHLS), Tshwane Academic Division (NHLS/TAD) in Pretoria from patients who were suspected to be infected with TB and had come for their first TB screening. The volume of each specimen differed as remnant samples were used; hence, $800 \mu \mathrm{L}$ of each specimen was used for the molecular assays. All remnant samples from persons suspected of having TB were included while those from doubtful sources, on treatment or had contaminations were discarded.

Sample processing. The 205 remnant samples were equally divided into three aliquots (volumes): one was processed by decontamination using $1 \%$ NALC-NaOH method for culture into MGIT 7H9 (BD, Sparks MD, USA); the second was used for the auramine smear microscopy; and the third sample ( $800 \mu \mathrm{L}$ per sample) was transferred into $1.8 \mathrm{~mL}$ PrimeStore (PS) molecular transport media (MTM) tubes (Longhorn Vaccines and 
Diagnostics LLC, USA) for molecular assays (Xpert and MTBDRplus) (Fig. 1). Each 1.8 mL PS-MTM tube contained $1 \mathrm{~mL}$ of PS-MTM. All samples were vortexed using the Vortex Genie 2 mixer (Scientific Industries Inc, USA) for 5-10 seconds. A volume of $800 \mu \mathrm{L}$ of specimen was aliquoted into the correctly labelled $1.8 \mathrm{~mL}$ PS-MTM tube, mixed by pipetting up and down thrice and the tip was discarded after use. Finally, the PrimeStore tubes were vortexed with the specimen using Vortex Genie 2 mixer (Scientific Industries Inc, USA) and stored at room temperature for later use. The specimens stored in PrimeStore were divided into three: one specimen $(200 \mu \mathrm{L})$ was used for DNA extraction for the LPA assay and the other two were used for the Xpert Ultra assay $(500 \mu \mathrm{L})$ and for the old Xpert G4 assay $(500 \mu \mathrm{L})$ according to manufacturer's instructions ${ }^{13,14}$.

Auramine smear microscopy. The second portion of the 205 specimens were subjected to the auramine smear microscopy by fixing the specimens unto slides, applying rhodamine auramine to the fixed slide for 5 minutes, and then rinsing it, applying the decolorizing agent, acid-alcohol, and then rinsing it, applying potassium permanganate for 2 minutes and then rinsing, drying and observing the slide with a fluorescent microscope as already described (Wanger et al., 2017).

GeneXpert MTB/RIF assays. The GeneXpert assays (Ultra and G4) were conducted on all 205 (125 pulmonary and 80 extra-pulmonary) samples. A $1.0 \mathrm{~mL}$ of GeneXpert MTB/RIF assay sample reagent (Cepheid Inc, USA) was added to $500 \mu \mathrm{L}$ of each specimen (already mixed with the PS-MTM) using a sterile pipette (Tang et al., 2017).

DNA extraction. DNA was extracted from all 205 (125 pulmonary and 80 extra-pulmonary) specimens using PrimeXtract extraction kits (Long Horn Vaccines and Diagnostics, USA). A volume of $200 \mu \mathrm{L}$ of $100 \%$ ethanol, $200 \mu \mathrm{L}$ lysis buffer and $200 \mu \mathrm{L}$ specimen (already mixed with the PS-MTM) were transferred into a $1.5 \mathrm{~mL}$ micro-centrifuge tube followed by thorough mixing and subsequent centrifugation. The entire supernatant was then pipetted into a micro extraction column, where it was centrifuged for $1 \mathrm{~min}$ at $13,000 \mathrm{x} \mathrm{g}$, and the flow-through material was discarded. A wash buffer $(200 \mu \mathrm{L})$ was pipetted into the extraction column and centrifuged for $1 \mathrm{~min}$ at $13000 \mathrm{xg}$, followed by further addition of wash buffer $(200 \mu \mathrm{L})$ to the extraction column, and subsequent centrifuging as described above, discarding the flow through material. The total $M$. $t b$ DNA was eluted by centrifuging for $1 \mathrm{~min}$ at maximum speed using $50 \mu \mathrm{L}$ of elution solution preheated at $75^{\circ} \mathrm{C}$. The DNA was stored at $-20^{\circ} \mathrm{C}$ for future use.

The GenoType MTBDRplus LPA test was conducted according to the manufacturer's instructions. Briefly, amplification reactions were undertaken using a $35 \mu \mathrm{L}$ primer nucleotide mix, $10 \mu \mathrm{L}$ polymerase mix (Hain Lifescience, Germany), and $5 \mu \mathrm{L}$ of genomic DNA. PCR and hybridization were performed and interpreted as per the manufacturer's instructions using the GTBlot and GenoScan machines (Hain Lifescience, Germany).

Sensitivity, specificity, and negative and positive predictive values calculations. Sensitivity, specificity, negative-predictive values (NPV) and positive-predictive values (PPV) were calculated using equations reported by Carvajal and Rowe $(2010)^{15}$.

Ethical approval. Ethical approval was obtained from the Human Research Ethics Committee (UPHREC) at the Faculty of Health Sciences, University of Pretoria (Ethics Reference Number: 153/2018) before the study commenced. All protocols and consent forms were executed according to the agreed ethical approval terms and conditions. All clinical samples were obtained from a reference laboratory and not directly from patients, who agreed (through informed consent forms) to our using their specimens for this research. The guidelines stated by the Declaration of Helsinki for involving human participants were followed in the study.

\section{Results}

MGIT 960 culture. Out of the 205 samples, 63/205 (30.73\%) were culture positive, 139/205 (67.80\%) were culture negative and three (1.46\%) were contaminated. Fifty out of 125 pulmonary were culture positive while 72 were culture negative; three pulmonary specimen were contaminated. Furthermore, 13 extra-pulmonary samples $(\mathrm{n}=80)$ were culture positive while 67 were culture negative (Table 1$)$

Auramine smear microscopy. The auramine smear microscopy resulted in 59/205 (28.78\%) smear positives and 146/205 (70.73\%) smear negatives. In pulmonary specimens, 51/125 (40.80\%) were smear positive and $74 / 125(59.20 \%)$ were smear negative while in extra-pulmonary specimens, $8 / 80$ (10.00\%) were smear positive and $72 / 80(90.00 \%)$ were smear negative.

GeneXpert MTB/RIF assays. For Xpert Ultra, 76/125 (60.80\%) specimens were positive for TB and 47/125 $(37.60 \%)$ were negative in pulmonary samples, with two (2/125) samples being invalid while $43 / 80(53.75 \%)$ specimens were positive for TB and $37 / 80(46.25 \%)$ were negative in extra-pulmonary samples.

For the old XpertG4, 49/125 (39.20\%) of specimens were TB-positive and 75/125 (60.00\%) were negative, with one (1/125) having invalid results in pulmonary samples while $8 / 80(10.00 \%)$ specimens were positive for TB and $71 / 80(88.75 \%)$ were negative, one of which had invalid results in extra-pulmonary specimens.

GenoType MTBDRplus LPA assay. For LPA, 107/125 (85.60\%) specimens were TB-positive and 18/125 (14.40\%) were TB-negative in pulmonary samples while 59/80 (73.75\%) specimens were TB-positive, and 21/80 (26.25\%) were TB-negative in extra-pulmonary samples (Table 1).

Sensitivity, specificity, PPV and NPV. For all the tests, i.e. auramine smear microscopy, Xpert Ultra, Xpert G4 and LPA), the sensitivities were higher in pulmonary samples than extra-pulmonary samples. A similar trend was observed in the specificity of Xpert Ultra, for which pulmonary samples had a higher specificity 


\begin{tabular}{|c|c|c|c|c|}
\hline Category & $\begin{array}{l}\text { Pulmonary } \\
\text { samples }\end{array}$ & $\begin{array}{l}\text { Extra-pulmonary } \\
\text { samples }\end{array}$ & Total & $\begin{array}{l}\text { Cumulative } \\
\text { total }\end{array}$ \\
\hline \multicolumn{5}{|l|}{ Specimen type } \\
\hline Sputum & 117 & NA & 117 & 117 \\
\hline abscess & $\mathrm{NA}^{*}$ & 4 & 4 & 121 \\
\hline csf & NA & 3 & 3 & 124 \\
\hline Fluid/aspirate & NA & 18 & 18 & 142 \\
\hline swab (superficial) & NA & 4 & 4 & 146 \\
\hline Tissue & NA & 15 & 15 & 161 \\
\hline tracheal/aspirate & 8 & NA & 8 & 169 \\
\hline urine & NA & 36 & 36 & 205 \\
\hline Total & 125 & 80 & 205 & 205 \\
\hline \multicolumn{5}{|l|}{ Culture results } \\
\hline Culture Contaminated & 3 & 0 & 3 & 3 \\
\hline Culture positive & 50 & 13 & 63 & 66 \\
\hline Culture negative & 72 & 67 & 139 & 205 \\
\hline Total & 125 & 80 & 205 & 205 \\
\hline \multicolumn{5}{|l|}{ Auramine smear microscopy results } \\
\hline Smear (auramine) positive & 51 & 8 & 59 & 59 \\
\hline Smear (auramine) negative & 74 & 72 & 146 & 205 \\
\hline Total & 125 & 80 & 205 & 205 \\
\hline \multicolumn{5}{|l|}{ Xpert Ultra results } \\
\hline Xpert Ultra positive (TB detected) & 76 & 43 & 119 & 119 \\
\hline Xpert Ultra negative (TB undetected) & 47 & 37 & 84 & 203 \\
\hline Xpert Ultra Invalid & 2 & 0 & 2 & 205 \\
\hline Total & 125 & 80 & 205 & 205 \\
\hline \multicolumn{5}{|l|}{ Ultra G4 results } \\
\hline Xpert G4 positive (TB detected) & 49 & 8 & 57 & 57 \\
\hline Xpert G4 negative (TB undetected) & 75 & 71 & 146 & 203 \\
\hline Xpert G4 error & 1 & 0 & 1 & 204 \\
\hline Xpert G4 invalid & 0 & 1 & 1 & 205 \\
\hline Total & 125 & 80 & 205 & 205 \\
\hline \multicolumn{5}{|l|}{ LPA results } \\
\hline LPA positive (TB detected) & 107 & 59 & 166 & 166 \\
\hline LPA negative (TB undetected) & 18 & 21 & 39 & 205 \\
\hline Total & 125 & 80 & 205 & 205 \\
\hline \multicolumn{5}{|c|}{ Breakdown of results under culture and smear microscopy categorisation } \\
\hline Smear negative (SN)-Culture negative (CN) & 65 & 66 & 131 & 131 \\
\hline Smear positive (SP)-Culture positive (CP) & 41 & 7 & 48 & 179 \\
\hline SN-CP & 9 & 6 & 15 & 194 \\
\hline SP-CN & 7 & 1 & 8 & 202 \\
\hline Total & $122 *$ & 80 & $202^{*}$ & $202 *$ \\
\hline
\end{tabular}

Table 1. General characteristics of specimens used in evaluating the smear microscopy, Xpert G4, Xpert Ultra and LPA assays.

than extra-pulmonary specimens: $58.57 \%$ and $49.25 \%$ respectively. However, the specificities of auramine smear microscopy, Xpert G4 and LPA were higher in extra-pulmonary samples than in pulmonary specimens (Table 2).

The negative predictive value (NPV) was $100.00 \%$ for all tests and samples within this category while auramine smear microscopy had a specificity of $100.00 \%$ for all samples within this category. The specificity of Xpert G4 was very high, between $98.46 \%$ and $98.48 \%$ for both extra-pulmonary and pulmonary samples. The specificity of Xpert Ultra was between $50.00 \%$ and $53.49 \%$ while that of LPA was below $26 \%$ for both pulmonary and extra-pulmonary samples within this category (Table 3 ).

In smear positive-culture positive (SP-CP) samples, sensitivity of auramine, Xpert Ultra, Xpert G4 and LPA was between $92-100 \%$ while the specificity and NPV were $0.00 \%$ for all the assays; PPV was $100 \%$ for all assays (Table 3). In smear-negative culture-positive (SN-CP) samples, specificity and NPV were both $0.00 \%$ for all samples and tests while PPV for auramine was $0.00 \%$ for both pulmonary and extra-pulmonary samples. However, the PPV for auramine Xpert Ultra, Xpert G4 and LPA was $100.00 \%$ for all samples, but that of Xpert G4 was $0.00 \%$ for extra-pulmonary while that of Xpert Ultra and LPA was $100.00 \%$ for extra-pulmonary samples. The sensitivity for auramine was $0.00 \%$ for all samples and less than $50.00 \%$ for all samples for Xpert Ultra, Xpert G4 and LPA, except for LPA sensitivity in extra-pulmonary samples, which was $66.67 \%$ (Table 3 ). 


\begin{tabular}{|c|c|c|c|c|}
\hline Parameter & Auramine & Xpert Ultra & Xpert G4 & LPA \\
\hline \multicolumn{5}{|l|}{ Pulmonary samples } \\
\hline True Positive & 41 & 44 & 39 & 40 \\
\hline True Negative & 65 & 41 & 65 & 8 \\
\hline False Positive & 7 & 29 & 7 & 64 \\
\hline False Negative & 9 & 6 & 10 & 10 \\
\hline Total & 122 & $120^{\ddagger}$ & $121^{\S}$ & 122 \\
\hline Sensitivity (CI), \% & $82,00(68,56-91,42)$ & $88,00(75,69-95,47)$ & $79,59(65,66-89,76)$ & $80,00(66,28-89,97)$ \\
\hline Specificity (CI), \% & $90,28(80,99-96,00)$ & $58,57(46,17-70,23)$ & $90,28(80,99-96,00)$ & $11,11(4,92-20,72)$ \\
\hline Positive predictive value (CI), \% & $85,42(74,11-92,30)$ & $60,27(53,00-67,12)$ & $84,78(73,10-91,95)$ & $38,46(34,73-42,33)$ \\
\hline Negative predictive value (CI), \% & $87,84(79,91-92,91)$ & $87,23(75,87-93,69)$ & $86,67(78,81-91,91)$ & $44,44(25,35-65,33)$ \\
\hline \multicolumn{5}{|l|}{ Extra-pulmonary samples } \\
\hline True Positive & 7 & 9 & 6 & 9 \\
\hline True Negative & 66 & 33 & 65 & 17 \\
\hline False Positive & 1 & 34 & 2 & 50 \\
\hline False Negative & 6 & 4 & 6 & 4 \\
\hline Total & 80 & 80 & $79+1^{* * *}$ & 80 \\
\hline Sensitivity (CI), \% & $53,85(25,13-80,78)$ & $69,23(38,57-90,91)$ & $50,00(21,09-78,91)$ & $69,23(38,57-90,91)$ \\
\hline Specificity (CI), \% & $98,51(91,96-99,96)$ & $49,25(36,82-61,76)$ & $97,01(89,63-99,64)$ & $25,37(15,53-37,49)$ \\
\hline Positive predictive value (CI), \% & $87,50(48,41-98,12)$ & $20,93(14,66-28,97)$ & $75,00(40,63-92,93)$ & $15,25(10,88-20,98)$ \\
\hline Negative predictive value (CI), \% & $91,67(85,94-95,19)$ & $89,19(77,89-95,08)$ & $91,55(86,00-95,03)$ & $80,95(63,04-91,37)$ \\
\hline
\end{tabular}

Table 2. Relative diagnostic efficiencies of smear microscopy, Xpert Ultra, Xpert G4 and LPA tests in detecting Mycobacterium tuberculosis from pulmonary and extra-pulmonary samples. ${ }^{\dagger} 3$ Samples were contaminated and discarded; adding the 3 contaminated isolates equals 205 . ${ }^{\ddagger} 2$ Isolates were invalid. Adding them will amount to $122 .{ }^{\S} 1$ Isolate was in error, adding it will make up to $122 .{ }^{* *} 1$ isolate was invalid. Adding it will amount to 80 ; \# 1 isolate was in error, adding it will make up to 122 .

In smear-positive culture-negative (SP-CN) samples, the sensitivity and PPV and NPV were $0.00 \%$ for all four tests in both extra-pulmonary and pulmonary specimens. However, a 100.00\% NPV was obtained for Xpert Ultra and Xpert G4 in the combined (both pulmonary and extra-pulmonary) and pulmonary samples (Table 3). Specificity was $0.00 \%$ for all four tests for extra-pulmonary samples, $0.00 \%$ for auramine and LPA for combined and pulmonary samples. Specificity of Xpert G4 and Ultra were both $12.50 \%$ for combined samples, and $14.21 \%$ and $14.29 \%$ for pulmonary samples respectively (Table 3; Supplementary Dataset).

Rifampicin resistance was detected in 19 isolates, with 16 and one being detected by Xpert Ultra in sputum from SN-CN and SN-CP samples respectively. While the 16 RIF-resistant isolates in SN-CN samples were detected only by Xpert Ultra, the single RIF-resistant isolates in SN-CP samples were detected by both Xpert Ultra and G4. LPA detected two RIF-resistant isolates, which was not confirmed by either Xpert Ultra or Xpert G4, in a single tissue SN-CP sample and in a sputum SP-CP sample (Supplementary Dataset). Other antibiotics resistance conferring-mutations were not determined.

\section{Discussion}

In this study, we compared the performance of the new Xpert Ultra assay with the old Xpert G4 assay, the LPA and auramine smear microscopy for detecting TB in pulmonary and EPTB clinical specimens. All these tests are used as initial rapid diagnostic tests in individuals suspected of having $\mathrm{TB}^{12}$. Our results show that the new Xpert Ultra assay has the highest sensitivity (88\%) for detecting TB among both pulmonary and EPTB clinical specimens (Table 2), followed by the auramine smear microscopy (82\%), LPA (80\%) and the old Xpert G4 (79.59\%), which had the lowest sensitivity. These results are consistent with the results from recent reports, which showed that Xpert Ultra had a superior sensitivity for TB case detection ${ }^{10,16}$. Chakravorty et al. (2017), reported an overall sensitivity of $87.50 \%$ for the Xpert Ultra versus a sensitivity of $81.00 \%$ for the old Xpert. However, this high sensitivity by the Xpert Ultra led to a lower specificity of $58.57 \%$ in pulmonary samples and $49.25 \%$ in extra-pulmonary samples as reported in other studies ${ }^{10,16}$. The low specificity could be due to the high rate of Xpert Ultra false positives in mostly pulmonary specimens and the fact that Xpert Ultra has increased limits of detection as compared to other assays ${ }^{11,16}$. Previous TB episodes may also cause false positives due to dead DNA in the sputum ${ }^{11,16}$.

In terms of specificity however, the auramine smear microscopy was higher in both pulmonary and EPTB specimens i.e., $90.28 \%$ and $98.51 \%$ respectively, than the Xpert G4 (90.28\% and $97.01 \%$ respectively), Xpert Ultra ( $58.57 \%$ and $49.25 \%$ respectively), and the LPA ( $11.11 \%$ and $25.37 \%$ respectively), which recorded the lowest specificity among all the tests. It is evident from these results that all the tests are more sensitive to $M$. $t b$ in pulmonary specimens than in EPTB specimens. Except for the Xpert Ultra, all the tests were more specific in detecting the absence of TB in EPTB specimens than in pulmonary clinical samples. The very close sensitivity of the auramine smear microscopy to the Xpert Ultra assay, particularly in pulmonary samples, and its better specificity in detecting the presence and absence of TB in both pulmonary and extra-pulmonary clinical specimens make it a good alternative to these nucleic acid tests. Coupled with its lower costs, lesser skills required and easier 


\begin{tabular}{|c|c|c|c|c|c|c|c|c|c|c|c|c|}
\hline \multirow[b]{2}{*}{ Parameter } & \multicolumn{4}{|c|}{ All samples (both pulmonary and extra-pulmonary) } & \multicolumn{4}{|c|}{ Pulmonary samples } & \multicolumn{4}{|c|}{ Extra-pulmonary samples } \\
\hline & Auramine & \begin{tabular}{|l|} 
Xpert \\
Ultra
\end{tabular} & Xpert G4 & LPA & Auramine & \begin{tabular}{|l|} 
Xpert \\
Ultra
\end{tabular} & Xpert G4 & LPA & Auramine & \begin{tabular}{|l|} 
Xpert \\
Ultra
\end{tabular} & Xpert G4 & LPA \\
\hline & \multicolumn{4}{|c|}{ Smear Negative-Culture Negative (SN-CN) } & \multicolumn{4}{|c|}{ Smear Negative-Culture Negative (SN-CN) } & \multicolumn{4}{|c|}{ Smear Negative-Culture Negative (SN-CN) } \\
\hline True Positive & 0 & 0 & 0 & 0 & 0 & 0 & 0 & 0 & 0 & 0 & 0 & 0 \\
\hline True Negative & 131 & 73 & 129 & 25 & 65 & 40 & 64 & 8 & 66 & 33 & 65 & 17 \\
\hline False Positive & 0 & 56 & 2 & 106 & 0 & 23 & 1 & 57 & 0 & 33 & 1 & 49 \\
\hline False Negative & 0 & 0 & 0 & 0 & 0 & 0 & 0 & 0 & 0 & 0 & 0 & 0 \\
\hline Total & 131 & $129^{\dagger \dagger}$ & 131 & 131 & 65 & $63^{1 \dagger}$ & 65 & 65 & 66 & 66 & 66 & 66 \\
\hline Sensitivity (CI), \% & 0 & 0 & 0 & 0 & 0 & 0 & 0 & 0 & 0 & 0 & 0 & 0 \\
\hline Specificity $(\mathrm{CI}), \%$ & $\begin{array}{l}100,00 \\
(97,22- \\
100,00) \\
\end{array}$ & \begin{tabular}{|l|}
56,59 \\
$(47,58-$ \\
$65,29)$ \\
\end{tabular} & \begin{tabular}{|l|}
98,47 \\
$(94,59-$ \\
$99,81)$ \\
\end{tabular} & \begin{tabular}{|l|}
19,08 \\
$(12,75-$ \\
$26,87)$
\end{tabular} & $\begin{array}{l}100,00 \\
(94,48- \\
100,00)\end{array}$ & \begin{tabular}{|l|}
63,49 \\
$(50,40-$ \\
$75,27)$
\end{tabular} & \begin{tabular}{|l|}
98,46 \\
$(91,72-$ \\
$99,96)$ \\
\end{tabular} & $\begin{array}{l}12,31 \\
(5,47- \\
22,82)\end{array}$ & $\begin{array}{l}100,00 \\
(94,56- \\
100,00)\end{array}$ & \begin{tabular}{|l|}
50,00 \\
$(37,43-$ \\
$62,57)$ \\
\end{tabular} & \begin{tabular}{|l|}
98,48 \\
$(91,84-$ \\
$99,96)$
\end{tabular} & \begin{tabular}{|l|}
25,76 \\
$(15,78-$ \\
$38,01)$
\end{tabular} \\
\hline $\begin{array}{l}\text { Positive predictive } \\
\text { value }(\mathrm{CI}), \%\end{array}$ & 0 & 0 & 0 & 0 & 0 & 0 & 0 & 0 & 0 & 0 & 0 & 0 \\
\hline \multirow[t]{2}{*}{$\begin{array}{l}\text { Negative predictive } \\
\text { value }(\mathrm{CI}), \%\end{array}$} & 100 & 100 & 100 & 100 & 100 & 100 & 100 & 100 & 100 & 100 & 100 & 100 \\
\hline & \multicolumn{4}{|c|}{ Smear Positive-Culture Positive (SP-CP) } & \multicolumn{4}{|c|}{ Smear Positive-Culture Positive (SP-CP) } & \multicolumn{4}{|c|}{ Smear Positive-Culture Positive (SP-CP) } \\
\hline True Positive & 48 & 47 & 43 & 43 & 41 & 40 & 37 & 38 & 7 & 7 & 6 & 5 \\
\hline True Negative & 0 & 0 & 0 & 0 & 0 & 0 & 0 & 0 & 0 & 0 & 0 & 0 \\
\hline False Positive & 0 & 0 & 0 & 0 & 0 & 0 & 0 & 0 & 0 & 0 & 0 & 0 \\
\hline False Negative & 0 & 1 & 3 & 5 & 0 & 1 & 3 & 3 & 0 & 0 & 0 & 2 \\
\hline Total & 48 & 48 & $46^{\S 5}$ & 48 & 41 & 41 & $40+1^{* * *}$ & 41 & 7 & 7 & $6+1^{t+t}$ & 7 \\
\hline Sensitivity (CI), \% & $\begin{array}{l}100,00 \\
(92,60- \\
100,00) \\
\end{array}$ & \begin{tabular}{|l|}
97,92 \\
$(88,93-$ \\
$99,95)$ \\
\end{tabular} & \begin{tabular}{|l|}
93,48 \\
$(82,10-$ \\
$98,63)$ \\
\end{tabular} & \begin{tabular}{|l|}
89,58 \\
$(77,34-$ \\
$96,53)$ \\
\end{tabular} & \begin{tabular}{l|}
100,00 \\
$(91,40-$ \\
$100,00)$ \\
\end{tabular} & \begin{tabular}{|l|}
97,56 \\
$(87,14-$ \\
$99,94)$ \\
\end{tabular} & \begin{tabular}{|l}
92,50 \\
$(79,61-$ \\
$98,43)$ \\
\end{tabular} & \begin{tabular}{|l|}
92,68 \\
$(80,08-$ \\
$98,46)$ \\
\end{tabular} & $\begin{array}{l}100,00 \\
(59,04- \\
100,00) \\
\end{array}$ & \begin{tabular}{|l|}
100,00 \\
$(59,04-$ \\
$100,00)$ \\
\end{tabular} & $\begin{array}{l}100,00 \\
(54,07- \\
100,00) \\
\end{array}$ & \begin{tabular}{|l|}
62,50 \\
$(24,49-$ \\
$91,48)$ \\
\end{tabular} \\
\hline Specificity (CI), \% & 0 & 0 & 0 & 0 & 0 & 0 & 0 & 0 & 0 & 0 & 0 & 0 \\
\hline $\begin{array}{l}\text { Positive predictive } \\
\text { value }(\mathrm{CI}), \%\end{array}$ & 100 & 100 & 100 & 100 & 100 & 100 & 100 & 100 & 100 & 100 & 100 & 100 \\
\hline \multirow[t]{2}{*}{$\begin{array}{l}\text { Negative predictive } \\
\text { value }(\mathrm{CI}), \%\end{array}$} & 0 & 0 & 0 & 0 & 0 & 0 & 0 & 0 & 0 & 0 & 0 & 0 \\
\hline & \multicolumn{4}{|c|}{ Smear Negative-Culture Positive (SN-CP) } & \multicolumn{4}{|c|}{ Smear Negative-Culture Positive (SN-CP) } & \multicolumn{4}{|c|}{ Smear Negative-Culture Positive (SN-CP) } \\
\hline True Positive & 0 & 6 & 2 & 6 & 0 & 4 & 2 & 2 & 0 & 2 & 0 & 4 \\
\hline True Negative & 0 & 0 & 0 & 0 & 0 & 0 & 0 & 0 & 0 & 0 & 0 & 0 \\
\hline False Positive & 0 & 0 & 0 & 0 & 0 & 0 & 0 & 0 & 0 & 0 & 0 & 0 \\
\hline False Negative & 15 & 9 & 13 & 9 & 9 & 5 & 7 & 7 & 6 & 4 & 6 & 2 \\
\hline Total & 15 & 15 & 15 & 15 & 9 & 9 & 9 & 9 & 6 & 6 & 6 & 6 \\
\hline Sensitivity (CI), \% & $\begin{array}{l}0,00(0,00- \\
21,80)\end{array}$ & \begin{tabular}{|l|}
40,00 \\
$(16,34-$ \\
$67,71)$ \\
\end{tabular} & $\begin{array}{l}13,33(1,66- \\
40,46)\end{array}$ & \begin{tabular}{|l|}
40,00 \\
$(16,34-$ \\
$67,71)$ \\
\end{tabular} & $\begin{array}{l}0,00(0,00- \\
33,63)\end{array}$ & \begin{tabular}{|l|}
44,44 \\
$(13,70-$ \\
$78,80)$ \\
\end{tabular} & $\begin{array}{l}22,22(2,81- \\
60,01)\end{array}$ & \begin{tabular}{l|}
22,22 \\
$(2,81-$ \\
$60,01)$
\end{tabular} & $\begin{array}{l}0,00(0,00- \\
45,93)\end{array}$ & $\begin{array}{l}33,33(4,33- \\
77,72)\end{array}$ & $\begin{array}{l}0,00(0,00- \\
45,93)\end{array}$ & \begin{tabular}{|l}
66,67 \\
$(22,28-$ \\
$95,67)$
\end{tabular} \\
\hline Specificity (CI), \% & 0 & 0 & 0 & 0 & 0 & 0 & 0 & 0 & 0 & 0 & 0 & 0 \\
\hline $\begin{array}{l}\text { Positive predictive } \\
\text { value (CI), \% }\end{array}$ & 0 & 100 & 100 & 100 & 0 & 100 & 100 & 100 & 0 & 100 & 0 & 100 \\
\hline \multirow[t]{2}{*}{$\begin{array}{l}\text { Negative predictive } \\
\text { value }(\mathrm{CI}), \%\end{array}$} & 0 & 0 & 0 & 0 & 0 & 0 & 0 & 0 & 0 & 0 & 0 & 0 \\
\hline & \multicolumn{4}{|c|}{ Smear Positive-Culture Negative (SP-CN) } & \multicolumn{4}{|c|}{ Smear Positive-Culture Negative (SP-CN) } & \multicolumn{4}{|c|}{ Smear Positive-Culture Negative (SP-CN) } \\
\hline True Positive & 0 & 0 & 0 & 0 & 0 & 0 & 0 & 0 & 0 & 0 & 0 & 0 \\
\hline True Negative & 0 & 1 & 1 & 0 & 0 & 1 & 1 & 0 & 0 & 0 & 1 & 0 \\
\hline False Positive & 8 & 7 & 7 & 8 & 7 & 6 & 6 & 7 & 1 & 1 & 1 & 1 \\
\hline False Negative & 0 & 0 & 0 & 0 & 0 & 0 & 0 & 0 & 0 & 0 & 0 & 0 \\
\hline Total & 8 & 8 & 8 & 8 & 7 & 7 & 7 & 7 & 1 & 1 & 1 & 1 \\
\hline Sensitivity (CI), \% & 0 & 0 & 0 & 0 & 0 & 0 & 0 & 0 & 0 & 0 & 0 & 0 \\
\hline Specificity (CI), \% & $\begin{array}{l}0,00(0,00- \\
36,94)\end{array}$ & $\begin{array}{l}12,50(0,32- \\
52,65)\end{array}$ & $\begin{array}{l}12,50(0,32- \\
52,65)\end{array}$ & $\begin{array}{l}0,00(0,00- \\
36,94)\end{array}$ & $\begin{array}{l}0,00(0,00- \\
40,96)\end{array}$ & $\begin{array}{l}14,29 \\
(0,36- \\
57,87)\end{array}$ & $\begin{array}{l}14,29(0,36- \\
57,87)\end{array}$ & $\begin{array}{l}0,00(0,00- \\
40,96)\end{array}$ & $\begin{array}{l}0,00(0,00- \\
97,50)\end{array}$ & $\begin{array}{l}0,00(0,00- \\
97,50)\end{array}$ & $\begin{array}{l}0,00(0,00- \\
97,50)\end{array}$ & $\begin{array}{l}0,00 \\
(0,00- \\
97,50)\end{array}$ \\
\hline $\begin{array}{l}\text { Positive predictive } \\
\text { value (CI), } \%\end{array}$ & 0 & 0 & 0 & 0 & 0 & 0 & 0 & 0 & 0 & 0 & 0 & 0 \\
\hline $\begin{array}{l}\text { Negative predictive } \\
\text { value }(\mathrm{CI}), \%\end{array}$ & 0 & 100 & 100 & 0 & 0 & 100 & 100 & 0 & 0 & 0 & 0 & 0 \\
\hline
\end{tabular}

Table 3. Breakdown of the relative diagnostic efficiencies of smear microscopy, Xpert Ultra, Xpert G4 and LPA tests in detecting Mycobacterium tuberculosis from pulmonary and extra-pulmonary samples under different culture and smear results categories. ${ }^{\dagger \dagger} 2$ Isolates were invalid. Adding them will amount to $131 .{ }^{\ddagger} 2$ Isolates were invalid. Adding them will amount to $65 .{ }^{\S} 2$ Isolates were invalid/error. Adding them will amount to 46 . ${ }^{* * *} 1$ Isolate was invalid/error. Adding it will amount to $41 .{ }^{\dagger \dagger} 1$ Isolate was invalid/error. Adding that will amount to 7. 
application in lower resource settings, our findings support the WHO's recommendation for the use of the smear microscopy in detecting TB.

Although highly sensitive, the poorer specificity of the Xpert Ultra is a cause for concern as it can lead to high rate of false positives and subsequent placement of such patients on very toxic TB regimens for at least six months. Thus, there is the need to supplement the Xpert Ultra with either culture or the smear microscopy, preferably the latter due to its rapidity and simplicity, to augment for the lower specificity of the Xpert Ultra. Nevertheless, it is also worthy to note that the culture assay, which was used as the gold standard in this study, cannot identify dead, dormant or non-viable $M$. $t b$ strains, a situation that can also lead to false positive results in NAATs that detect DNA in clinical samples. Although MGIT has an analytical sensitivity threshold of 10 bacilli (colony forming units per $\mathrm{ml}$ ), which is equivalent to Xpert Ultra, the decontamination step reduces the number of bacilli in the sputum. Thus, the MGIT culture is an imperfect gold standard and final diagnosis can be done with other tests (i.e. symptom screen, X-Ray, microscopy). This is a major limitation of the culture tests, making it difficult to provide a true specificity of NAATs when the former is used as a gold standard for evaluating the latter. However, it should be noted that the samples included in this study were from patients who had come for their first TB screening. As a result, there should not be a substantial number of dead $M$. $t b$ DNA in the specimens. Particularly when they had not been given any TB chemotherapy.

In addition, the findings support the use of pulmonary samples for diagnosing TB in suspected patients instead of EPTB ones as the difference in sensitivities for all the four tests between pulmonary and extra-pulmonary samples is substantially wide. It is notable, however, that certain conditions might make it impossible to obtain pulmonary samples for TB diagnosis, specifically from infants and children who cannot produce sputum. And for such cases, the Xpert Ultra and the LPA seem a better diagnostic option due to their higher sensitivity (69.23\%) than the other two tests (Table 2). The less than $100 \%$ sensitivity of the LPA for smear-positive samples could be due to the presence of novel resistance mechanisms that are undetectable by the LPA probes, inability of culture to detect dead or dormant $M$. $t b$ cells or the processing procedures used ${ }^{17}$.

The specificities and NPVs of all the tests were lowest when culture was positive (SP-CP and SN-CP samples) irrespective of the source of the sample: pulmonary or extra-pumonary. Furthermore, in smear positive-culture positive (SP-CP) samples, the sensitivity was even higher for all the tests, with auramine having the highest sensitivity (100\%) followed by Xpert ultra (97.92\%), Xpert G4 (93.48\%) and LPA (89.58\%). Notably, a low sensitivity and PPV were recorded by all the tests in SN-CN and SP-CN samples, albeit with variable specificities that were substantially higher in SN-CN samples (from $19.08 \%$ in LPA to $98.47 \%$ in Xpert G4) than in SP-CN samples (from $12.50 \%$ for both Xpert assays down to $0 \%$ for LPA). This shows that a joint absence of TB in both culture and smear microscopy enhances the veracity of the NAATs in predicting the absence of TB than when the two phenotypic tests disagree (in SP-CN samples), which was strongly shown in the Xpert G4 results (Table 3). Contrary to SP-CP samples, where both smear and culture agree and all four tests had higher sensitivies, SN-CP samples, for which smear and culture disagree, reported very low sensitivies for all four tests. These results are consistent with, albeit lower than, the results reported by Dorman et al. (2018) where a superior sensitivity of 63\% for Ultra was seen vs $44 \%$ for the Xpert G4 in SN-CP specimen.

The highest sensitivity of the auramine stain in culture-positive specimens is not surprising as smear positivity normally correlates well with quantitative growth of the TB bacteria ${ }^{18}$. Moreover, because NAATs detect DNA, which are undetectable by culture and auramine, NAATs are more likely to report positive results in specimens for which auramine and culture, which detects cells, are negative. Smear microscopy is still the baseline diagnostic test in resource-limited, high TB-burden countries, specifically on the African continent and South East Asia ${ }^{12}$. However, this is not the case in South Africa as it has rolled out the highest number of Xpert matches than any other country in the world ${ }^{1}$. Smear microscopy is well known for its high specificity, which makes it a reliable test if the specimen does not have the TB bacteria. Our results are therefore consistent with the ones reported in the literature ${ }^{18,19}$.

Rapid diagnosis of EPTB is challenging because of the paucibacillary nature of the disease, causing rare positive smear microscopy results and long incubation time for culture growth ${ }^{20}$. In this study, the Xpert Ultra's sensitivity was superior to the old Xpert and smear microscopy with pulmonary specimen, albeit the Xpert Ultra had the same sensitivity as the LPA (69.23\%) in EPTB specimens. Our results are consistent with results from two studies that have evaluated the performance of Xpert Ultra in EPTB samples ${ }^{11,21}$. This detection improvement in the Xpert Ultra in EPTB samples is due to the larger PCR chamber, the incorporation of two different multicopy targets (IS1081 and IS6110 insertion sequences), and the optimization of PCR and thermal-cycling parameters ${ }^{16}$. The WHO strongly recommends the use of the Xpert assays in EPTB samples such as the cerebrospinal fluid over traditional smear microscopy and culture.

Ninan et al. (2016) reported similar sensitivity rates (67\%) in smear negative samples for LPA in their study as observed herein with SN-CP specimens, with our LPA also showing a sensitivity of $66.67 \%{ }^{22}$. However our LPA sensitivity was a little lower than that reported by Meaza et al. ${ }^{23}$. Our Xpert sensitivity in this group of specimens was within the range reported by Perez-Risco et al. ${ }^{11}$, with sensitivities ranging from $100 \%$ to $0 \%$ for different EPTB specimen types. In this study's EPTB samples, smear microscopy demonstrated the highest specificity (98.51\%), followed by the Xpert G4 (97.01\%), Xpert Ultra (49.25\%) and LPA (25.37\%). The assays were all TB-negative in SN-CN extra-pulmonary specimens, which confirms that the patients were negative to TB but presented with other related disease symptoms that could complicate the differential diagnoses.

The Xpert Ultra detected more RIF resistance than the Xpert G4 and the LPA, which supports one of the reasons the Xpert Ultra assay was developed i.e., to overcome the limitations of the old Xpert assays with regards to differentiating certain silent mutations, improving the detection of RIF resistance in mixed infections, and avoiding false-positive results in detecting RIF resistance in paucibacillary specimens ${ }^{16}$.

The use of Primestore MTM (Longhorn Vaccines and Diagnostics, USA) to preserve the specimens used in this study also played a very big role in the high detection of TB in the three molecular assays used in this study. The 
PrimStore media has been shown to concentrate and enhance $M . t b$ detection at low concentrations when using the Xpert assays ${ }^{24}$. The PrimeStore MTM is optimized to inactivate/kill infectious biological pathogens, including Haemophilus influenzae, M. tb, Gram-Positive/Negative bacteria, and viruses (Daum et al., 2015). It disrupts/lyses lipid membranes and inactivates nucleases ${ }^{14}$. Moreover, PrimeStore MTM preserves and stabilizes RNA and DNA at ambient temperature and is compatible with manual and high throughput RNA/DNA isolation kits ${ }^{14}$.

Major limitations to this study includes the relatively smaller sample size, the absence of samples from totally healthy patients to serve as negative controls, the use of samples from a single reference laboratory (although this laboratory serves about 200 clinics and at least six academic hospitals in three provinces in South Africa), and the inability of the MGIT to identify dormant or dead cells. In addition, as primestore MTM was used to concentrate our DNA from all samples, we suspect that it could have given an advantage to the molecular assays in terms of sensitivity as low bacilli load in specimens can be killed during decontamination step in culture assays. Notably, the sensitivity and specificity values of the NAATs might be impacted by the MGIT, which was used as a gold standard; specifically, when positive samples are identified as negative by the culture (MGIT) due to low bacillary load. This could explain the lower specificity of the LPA and Xpert Ultra.

\section{Conclusion}

A significant diagnostic performance of the GeneXpert Ultra assay in the detection of TB and RIF resistance was observed, confirming its efficiency for rapid diagnosis of TB in either pulmonary or extra-pulmonary specimens. Nevertheless, caution is advised in interpreting or using the Xpert Ultra's specificity (TB-negative predictions) results clinically without a confirmation with culture or smear microscopy. The LPA showed relatively comparable diagnostic accuracy in detecting TB in all clinical specimens, albeit it was poorly specific than the Xpert Ultra; more studies are needed to confirm these findings on the two molecular assays using a better gold standard.

Received: 6 August 2019; Accepted: 28 October 2019;

Published online: 12 November 2019

\section{References}

1. WHO. Global Tuberculosis Report 2017. Who, doi:WHO/HTM/TB/2017.23 (2017).

2. Osei Sekyere, J., Reta, M. A., Maningi, N. E. \& Fourie, P. B. Antibiotic Resistance of Mycobacterium tuberculosis complex in Africa : A Systematic Review of Current Reports of Molecular Epidemiology, Mechanisms and Diagnostics. J. Infect., 0 https://doi.org/10.1016/j. jinf.2019.10.006, https://linkinghub.elsevier.com/retrieve/pii/S0163445319303160 (2019).

3. Churchyard, G. J. et al. Tuberculosis control in South Africa: Successes, challenges and recommendations. South African Med. J., https://doi.org/10.7196/SAMJ.7689 (2014).

4. Helb, D. et al. Rapid detection of Mycobacterium tuberculosis and rifampin resistance by use of on-demand, near-patient technology. J. Clin. Microbiol. 48, 229-237 (2010).

5. Theron, G. et al. Determinants of PCR performance (Xpert MTB/RIF), including bacterial load and inhibition, for TB diagnosis using specimens from different body compartments. Sci. Rep. 4 (2014).

6. Denkinger, C. M. et al. Xpert MTB/RIF assay for the diagnosis of extrapulmonary tuberculosis: A systematic review and metaanalysis. Eur. Respir. J. 44, 435-446 (2014).

7. Zeka, A. N., Tasbakan, S. \& Cavusoglu, C. Evaluation of the GeneXpert MTB/RIF assay for rapid diagnosis of tuberculosis and detection of rifampin resistance in pulmonary and extrapulmonary specimens. J. Clin. Microbiol. 49, 4138-4141 (2011).

8. Köser, C. U., Feuerriegel, S., Summers, D. K., Archer, J. A. C. \& Niemann, S. Importance of the genetic diversity within the Mycobacterium tuberculosis complex for the development of novel antibiotics and diagnostic tests of drug resistance. Antimicrobial Agents and Chemotherapy 56, 6080-6087 (2012).

9. Chakravorty, S. et al. Rapid, high-throughput detection of rifampin resistance and heteroresistance in Mycobacterium tuberculosis by use of sloppy molecular beacon melting temperature coding. J. Clin. Microbiol. 50, 2194-2202 (2012).

10. Dorman, S. E. et al. Xpert MTB/RIF Ultra for detection of Mycobacterium tuberculosis and rifampicin resistance: a prospective multicentre diagnostic accuracy study. www.thelancet.com/infection Lancet Infect Dis 18, 76-84 (2018).

11. Perez-Risco, D., Rodriguez-Temporal, D., Valledor-Sanchez, I. \& Alcaidea, F. Evaluation of the Xpert MTB/RIF Ultra Assay for Direct Detection of Mycobacterium tuberculosis Complex in Smear-Negative Extrapulmonary Samples. J. Clin. Microbiol. 56 (2018).

12. WHO. World Health Organization Model List of Essential In Vitro Diagnostics. (2018).

13. Maningi, N. E. et al. Multi- and Extensively Drug Resistant Mycobacterium tuberculosis in South Africa: a Molecular Analysis of Historical Isolates. J. Clin. Microbiol. 56, JCM.01214-17 (2018).

14. Daum, L. T. et al. A molecular transport medium for collection, inactivation, transport, and detection of Mycobacterium tuberculosis. Int. J. Tuberc. Lung Dis. 18, 847-849 (2014).

15. Carvajal, D. N. \& Rowe, P. C. Research and Statistics: Sensitivity, Specificity, Predictive Values, and Likelihood Ratios. Pediatr. Rev. 31, 511-513 (2010)

16. Chakravorty, S. et al. The new Xpert MTB/RIF ultra: Improving detection of Mycobacterium tuberculosis and resistance to Rifampin in an assay suitable for point-of-care testing. MBio 8 (2017).

17. Nathavitharana, R. R. et al. Accuracy of line probe assays for the diagnosis of pulmonary and multidrug-resistant tuberculosis: a systematic review and meta-analysis. Eur. Respir. J. 49 (2017).

18. Hooja, S. et al. Comparison of Ziehl Neelsen \& Auramine O staining methods on direct and concentrated smears in clinical specimens. Indian J. Tuberc. 58, 72-6 (2011).

19. Agrawal, M., Bajaj, A., Bhatia, V. \& Dutt, S. Comparative study of GeneXpert with ZN stain and culture in samples of suspected pulmonary tuberculosis. J. Clin. Diagnostic Res. 10, DC09-DC12 (2016).

20. Rahman, F. et al. Comparison of Different Microscopic Methods with Conventional TB Culture. Stamford J. Microbiol., https://doi. org/10.3329/sjm.vli1.9133 (1970)

21. Bahr, N. C. et al. Diagnostic accuracy of Xpert MTB/RIF Ultra for tuberculous meningitis in HIV-infected adults: a prospective cohort study. Lancet Infect. Dis., https://doi.org/10.1016/S1473-3099(17)30474-7 (2018).

22. Ninan, M. M., Gowri, M., Christopher, D. J., Rupali, P. \& Michael, J. S. The diagnostic utility of line probe assays for multidrugresistant tuberculosis. Pathog. Glob. Health 110, 194-199 (2016).

23. Meaza, A. et al. Evaluation of genotype MTBDRplus VER 2.0 line probe assay for the detection of MDR-TB in smear positive and negative sputum samples. BMC Infect. Dis. 17 (2017)

24. Daum, L. T. et al. Xpert ${ }^{\circledR}$ MTB/RIF detection of Mycobacterium tuberculosis from sputum collected in molecular transport medium. Int. J. Tuberc. Lung Dis. 20, 1118-1124 (2016). 


\section{Acknowledgements}

We would like to thank the staff at the National Health Laboratory Services/Tshwane Academic Division (NHLS/ TAD) for their assistance in collecting the samples. N.E.M. received research grants from the University of Pretoria, National Reseach Foundation and Medical Reseach Council of South Africa. N.E.M. received research grants from the University of Pretoria, National Reseach Foundation and South African Medical Reseach Council.

\section{Author contributions}

Study design (J.O.S. and N.E.M.); Laboratory work (N.M. and N.E.M.); Data analyses (J.O.S., L.M. and N.E.M.); Manuscript write-up (J.O.S. and N.E.M.); Critical review (All authors).

\section{Competing interests}

The authors declare no competing interests.

\section{Additional information}

Supplementary information is available for this paper at https://doi.org/10.1038/s41598-019-53086-5.

Correspondence and requests for materials should be addressed to N.E.M.

Reprints and permissions information is available at www.nature.com/reprints.

Publisher's note Springer Nature remains neutral with regard to jurisdictional claims in published maps and institutional affiliations.

Open Access This article is licensed under a Creative Commons Attribution 4.0 International License, which permits use, sharing, adaptation, distribution and reproduction in any medium or format, as long as you give appropriate credit to the original author(s) and the source, provide a link to the Creative Commons license, and indicate if changes were made. The images or other third party material in this article are included in the article's Creative Commons license, unless indicated otherwise in a credit line to the material. If material is not included in the article's Creative Commons license and your intended use is not permitted by statutory regulation or exceeds the permitted use, you will need to obtain permission directly from the copyright holder. To view a copy of this license, visit http://creativecommons.org/licenses/by/4.0/.

(C) The Author(s) 2019 